\title{
Cancer Chemoprevention with Korean Angelica: Active Compounds, Pharmacokinetics, and Human Translational Considerations
}

\author{
Junxuan L $\ddot{u ̈}^{1} \cdot$ Jinhui Zhang ${ }^{1} \cdot \mathrm{Li} \mathrm{Li}^{1,3} \cdot$ Cheng Jiang $^{1} \cdot$ Chengguo Xing $^{2}$
}

Published online: 19 April 2015

(C) Springer International Publishing AG 2015

\begin{abstract}
Angelica gigas Nakai (AGN) is a major medicinal herb used in Korea and several other Asian countries. Traditionally, its dried root has been used to treat anemia, pain, infection, and articular rheumatism, most often through boiling in water to prepare the dosage forms. AGN extract or AGN-containing herbal mixtures are sold in the USA and globally as dietary supplements for pain killing, memory enhancement, and postmenopausal symptom relief. Decursin (D) and its isomer decursinol angelate (DA) are the major chemicals in the alcoholic extracts of the root of AGN. The anti-cancer activity of AGN alcoholic extract has been established in a number of animal cancer models, including a transgenic model of prostate carcinogenesis. Cell culture structure-activity studies have uncovered distinct cellular and molecular effects of D and DA vs. their pyranocoumarin core decursinol (DOH) with respect to cancer cells and those associated with their microenvironment. Pharmacokinetic (PK) study by us and others in rodent models indicated that $\mathrm{DOH}$ is the major and rapid in vivo first pass liver metabolite of D and DA. Cognizant of metabolic differences among rodents and humans, we carried out a first-in-human PK study of D/DA to inform the translational relevance of efficacy and mechanism studies with rodent models. The combined use of rigorous animal
\end{abstract}

This article is part of the Topical Collection on Cancer Chemoprevention

Junxuan Lü

junxuan.lu@ttuhsc.edu

1 Department of Biomedical Sciences, School of Pharmacy, Texas Tech University Health Sciences Center, 1300 S. Coulter St, Amarillo, TX 79106, USA

2 Department of Medicinal Chemistry, College of Pharmacy, University of Minnesota, Minneapolis, MN 55455, USA

3 Present address: Center for Drug Evaluation and Research, Food and Drug Administration, Silver Spring, Maryland, USA tests and human PK studies can provide stronger scientific rationale to inform design and execution of translational studies to move AGN toward evidence-based herbal medicine.

Keywords Angelica gigas Nakai · Decursin · Decursinol · Decursinol angelate $\cdot$ Pharmacokinetics $\cdot$ Translational cancer research
Abbreviations
AGN Angelica gigas Nakai
D Decursin
DA Decursinol angelate
DOH Decursinol

\section{Introduction}

We have in a recent article systematically reviewed the anticancer and other bio-activities of Korean Angelica and its major pyranocoumarin compounds [1•]. In this paper, we will focus on recent developments concerning the anti-cancer activities and new knowledge concerning the pharmacokinetics (PK) and metabolism of decursin (D) and its isomer decursinol angelate (DA), not only in rodent models but also in humans. Readers are referred to our comprehensive review $[1 \cdot]$ and the original references therein for historical information and specific details.

\section{Korean Angelica gigas Nakai and Its Major Phytochemicals}

Korean A. gigas Nakai (AGN) (in Korean Cham Dang-gui) differs from other prominent medicinal Dang-gui species 
including Angelica sinensis (Chinese) and Angelica acutiloba (Japanese) by its possession of unique pyranocoumarins [1 $[$. Angelica roots are traditionally used to treat anemia, cold, pain, and other diseases as folk medicines and known to herbalists as "female ginseng." However, these traditional medicinal properties were mostly based on using boiling water to extract the active ingredients. Changing the extraction solvents can lead to the recovery of different chemicals and therefore novel medicinal and pharmacological properties. In fact, many pyranocoumarin compounds have been identified from the alcoholic extracts of AGN roots, which could not be extracted by water.

Chemical composition of plants of the Angelica genus has been extensively studied and reviewed $[1 \cdot 2]$. Pyranocoumarins are the major chemicals identified in the ethanolic or methanolic extracts of AGN. Decursin (D) (Fig. 1a), the most abundant, was first isolated from root of
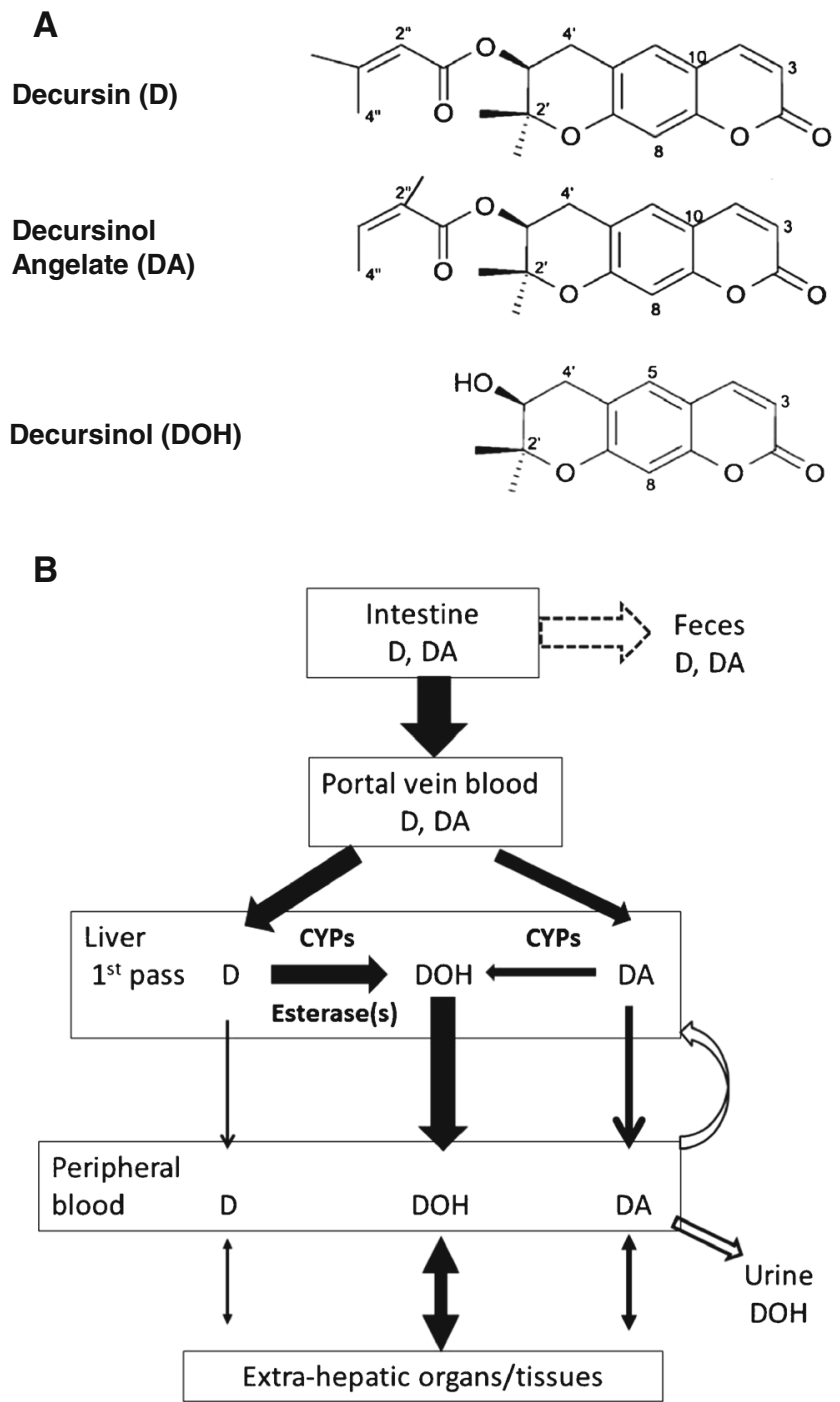

Fig. 1 a Chemical structures of decursin (D), decursinol angelate (DA), and decursinol (DOH). b Proposed metabolic schema of D and DA in rodents and humans after oral intake. CYP cytochrome $\mathrm{p} 450$
Angelica decursiva FR et SAV and later from AGN in the 1960s. Decursinol angelate (DA) (Fig. 1a) is a structural isomer of $\mathrm{D}$ on the side chain and about $60 \%$ as abundant as $\mathrm{D}$ in the root of AGN. The pyranocoumarin core decursinol (DOH) (Fig. 1a) can be identified with a much lower abundance, if at all, in AGN root. Additional chemicals structurally related to D include 4"-hydroxytigloyldecursinol, 4"-hydroxydecursin, ( 2 "S, 3"S)-epoxyangeloyldecursinol, and (2"R,3"R)epoxyangeloyldecursinol [1•]. The alcohol-extractable D/DA may constitute $3.0-8.2 \%$ of dried AGN root, depending on methods and herbal sources.

Classification based on a molecular basis in addition to morphology is very crucial to preventing adulteration of the dried roots of different Angelica species available in the herbal markets. Genetic differences among A. sinensis (Chinese), A. acutiloba (Japanese), and AGN (Korean) have been documented, including 5S-rRNA spacers [3]. They are phylogenetically divided into two clusters: the first group consisting of A. sinensis and the second group consisting of $A$. acutiloba and AGN [3]. Chemically, the contents of ferulic acid and Zligustilide in the root of $A$. sinensis were much higher than those of A. acutiloba and AGN [3]. Others have profiled the volatile components in Angelica species by gas chromatographic/mass spectrometry (GC/MS) $[4,5]$. GC/ MS profiling was used as a pattern recognition method for the quality control of AGN [6] with a principal component analysis (PCA) subsequently applied to 10 common peaks selected from each chromatogram. Profiling chemical components of AGN from different geographical origins using ${ }^{1} \mathrm{H}$ NMR and UPLC-MS analyses identified D, DOH, nodakenin, marmesin, and 7-hydroxy-6-(2R-hydroxy-3-methylbut-3ethyl)coumarin as major metabolites that contributed to the discrimination factor [7]. Taken together, D and DA are a robust identifying chemical characteristic of AGN.

\section{In Vivo Anti-cancer Activities of AGN Extracts}

We and collaborators reported in vivo inhibitory efficacy of AGN ethanol extracts on androgen-independent DU145 and PC-3 PCA xenografts [8] and in a primary prostate carcinogenesis model $[9 \bullet \cdot$. For the DU145 xenograft study, mice were treated with $100 \mathrm{mg} / \mathrm{kg}$ AGN in $1 \%$ Tween 80 by either gavage or intraperitoneal (i.p.) injection 7 days before tumor inoculation. By the end of experiment, treatment with AGN by i.p. injection and gavage suppressed the final tumor weight by 74 and $64 \%$, respectively. None of the treatment negatively affected the body weight of the mice. For the PC-3 xenograft study, treatment with $100 \mathrm{mg} / \mathrm{kg}$ AGN by i.p. injection started 4 days after tumor inoculation suppressed final tumor weight by $51 \%$. Biomarker analyses indicated that anti-proliferation, anti-angiogenesis, and apoptosis were likely 
involved in the in vivo suppressing effect of AGN against xenograft tumors.

We have recently shown that AGN inhibits the two lineages of carcinogenesis in the prostate of Transgenic Adenocarcinoma Mouse Prostate (TRAMP) model [9••]. In this study, male C57BL/6 TRAMP mice and wild type littermates were given a daily gavage ( $5 \mathrm{mg} / \mathrm{mouse}, \sim 200 \mathrm{mg} / \mathrm{kg}$, Monday-Friday) of AGN or $1 \%$ Tween 80 vehicle, beginning at 8 weeks of age (WOA). All mice were terminated at $24 \mathrm{WOA}$, unless earlier euthanasia was necessitated by large tumors. Whereas AGNtreated TRAMP mice decreased dorsolateral prostate growth by $30 \%(P=0.009)$, they developed fewer and smaller neuroendocrine-carcinomas (NE-Ca) $(0.12 \mathrm{~g} /$ mouse $)$ than vehicle-treated counterparts $(0.81 \mathrm{~g} /$ mouse, $P=0.037)$. Proteomic and transcriptomic analyses of banked NE-Ca suggest not only multiple cancer cell targeting actions of AGN but also significant impacts on the tumor microenvironments such as angiogenesis, inflammation, and immune surveillance. Prominent among upregulated gene categories by AGN were those involved in immune responses, prostate function/differentiation, ion transport, and tumor and proliferation suppressors $[9 \bullet \cdot]$. The immunity category of genes was particularly noteworthy. For example, defensin beta 50 (Defb50) was induced by AGN for 20-folds, and Defb1 for 5.7-folds. Defensin genes code for a family of 3-4 kDa polycationic peptides that act as bactericidal agents through disrupting bacterial membrane integrity. Clinical specimens had high frequencies of loss of Defb1 in malignant prostatic tissue, while high levels of expression were maintained in adjacent benign regions [10]. The finding was in agreement with the mouse model in which the expression of Defb1, Defb2, and Defb4 were decreased in TRAMP tumors [11, 12]. In addition, ectopical expression of Defb1 in DU145 and PC-3 PCA cells resulted in decreased cellular growth accomplished by cytolysis and caspase-mediated apoptosis [10]. Zinc alpha-2glycoprotein 1 (Azbp1) is a 41-kDa soluble protein synthesized by epithelial cells of many tissues including the prostate gland. Its expression was decreased in TRAMP prostate and tumor at $\mathrm{mRNA} /$ protein levels $[11,13]$. Recent findings indicated that absent/low Azgp1 expression was an independent predictor of recurrence in localized PCA after radical prostatectomy [14, 15]. Since Azgp1 has a major histocompatibility complex-1 (MHC1)-like fold in its structure, it might be involved in host immune response to tumor by antigen processing and presentation to exert its tumor suppressing effect. These and many other upregulated changes associated with immune responses suggest a potential promotion of immune surveillance by AGN treatment to inhibit NE-Ca growth.

Other notable changes suggested restoration by AGN of genes inactivated during TRAMP lesion progression. The prostate-specific proteins $\beta$-microseminoprotein $(\mathrm{Msmb})$ and serine peptidase inhibitor Kazal type 3 (Spink3) were 27.6 and 7.4-fold higher in AGN-treated NE-Ca, respectively, and we reported earlier their protein levels to be lower in TRAMP prostate than wild-type prostate [13]. GSTM1 and tropomyosin (Tpm2), which are downregulated in TRAMP prostate at protein level [13], and glutathione S-transferase, theta 3 were among tumor suppressor category of genes increased by AGN. The prostate epithelial-specific gene ventral prostate predominant 1 (Vpp1) was significantly induced by AGN treatment in the NE-Ca.

In terms of downregulated genes in the TRAMP NE-Ca, notable categories include neuron signaling and differentiation, oncogenes and proliferation, mast cells and inflammation, Wnt signaling/epithelial-mesenchymal-transition, embryonic morphogenesis, biosynthesis, cell adhesion, motility and invasion, and angiogenesis and hematopoiesis [9••]. The suppressed neuron signaling and differentiation genes could be expected because of the NE-Ca nature of the analyzed tissues and the significantly reduced NE-Ca burden. In particular, synaptotagmin IV (SYT4), known to play an important role in neurotransmitter secretion, was suppressed by AGN treatment as much as sevenfolds. The AGN-suppressed genes in other categories, such as oncogenes/oncofetal antigens and proliferation, Wnt signaling, biosynthesis, cell adhesion, motility and invasion, and angiogenesis and hemopoiesis, were consistent with them as potential contributors to the overall efficacy through not only direct action on cancer cells but also impact on their microenvironments. Several genes related to inflammatory mast cells, including mast cell carboxypeptidase A3 (Cpa3), proteases (Mcpt4; Mcpt6), mast cell chymases (Cma1; Cma2), and mast cell surface marker Fcerla (immunoglobulin epsilon receptor subunit alpha), were substantially suppressed by AGN treatment. Mast cells (MC) are granulocytic immune cells best known for their role in allergy and anaphylaxis. Pittoni et al. reported that in tumors from TRAM $\mathrm{P}$ mice on C57BL/6 background and human patients, MCs were specifically enriched and degranulated in areas of "well-differentiated" adenocarcinoma but not around "poorly differentiated" foci that coexist in the same tumors [16]. They showed that MCs promoted "well-differentiated" adenocarcinoma growth by providing MMP-9 but were dispensable for growth of "poorly differentiated" tumors [16]. Consistent with these findings, Morgenbesser et al. [17] and Kela et al. [11] showed that the expression of another MC surface marker c-kit was increased in TRAMP tumors. These findings suggested the role of MC and inflammation in prostatic carcinogenesis and might be suppressed by AGN to contribute to inhibition of NE-Ca growth.

In work to be published in full length, we compared AGN vs. equimolar intake of D/DA in the TRAMP model [18]. Experimentally, three cohorts of male C57BL/6 TRAMP mice (35-36 mice per group) were gavage-treated with a new vehicle (ethanol: PEG400: Tween 80: $5 \%$ glucose $=3: 6: 1: 20$ ), AGN ( $5 \mathrm{mg} / \mathrm{mouse}$ ) or equimolar D/DA ( $3 \mathrm{mg} /$ mouse) from 8 weeks of age (WOA). Mice were euthanized at either 16 WOA or 28 WOA unless large tumors necessitated earlier 
sacrifice. The results show that the growth of the TRAMP DLP lobes in AGN- and D/DA-treated mice were inhibited by 66 and $61 \%$, respectively (ANOVA, $P<0.0001$ ), from vehicle-control mice by $16 \mathrm{WOA}$. By $28 \mathrm{WOA}$, the inhibition of TRAMP DLP growth was 67 and $72 \%$, respectively (ANOVA, $P<0.001$ ). Whereas AGN and D/DA decreased the burden of NE-Ca, AGN improved survival of tumorbearing mice, but D/DA did not. In summary, AGN extract and equimolar intake of D/DA inhibited epithelial lineage lesions to identical extent; the extract was superior to D/DA at suppressing NE-carcinogenesis. Ongoing experiments have focused on (1) testing whether D/DA-hydrolysis product DOH mediates the TRAMP epithelial targeting inhibitory action and (2) identifying non-pyranocoumarin compounds in AGN extract that inhibit NE-carcinogenesis.

\section{Anti-cancer Activities of D, DA, and DOH}

Cell culture models have been primarily used as screening tools as well as cellular targets for studying potential anticancer activities and "mechanisms" of these compounds. Organ sites included prostate, breast, lung, colon, bladder, sarcoma, and blood cancers and have been extensively reviewed in our recent article [1•]. Here, we will focus on those organ sites with available in vivo efficacy information: prostate cancer, lung cancer, colon cancer, and sarcoma.

\section{Prostate Cancer}

Our group first identified D from AGN ethanol extract as a novel anti-androgen receptor (AR) compound with an $\mathrm{IC}_{50}$ of $1.3 \mu \mathrm{M}$ upon $48 \mathrm{~h}$ of exposure in the AR-dependent human prostate cancer cell line LNCaP measured by prostate-specific antigen (PSA) secretion [19]. Subsequently, we reported the anti-AR effect of D, DA, and DOH in terms of their structureactivity relationship [20]. Decursin and DA inhibited PSA levels in a dose-dependent manner with $\mathrm{IC}_{50}$ to be $1 \sim 1.3 \mu \mathrm{M}$ and suppressed AR levels through proteasomal degradation and blocked androgen-stimulated AR nuclear translocation. In addition, D and DA lacked the agonist activity of AR antagonist drug bicalutamide in the absence of androgen and were more potent than bicalutamide for suppressing androgen-stimulated cell growth [19]. On the other hand, the effect of DOH on PSA expression is biphasic, inhibiting PSA at lower micromolar range yet stimulating at higher concentrations [20]. In a high-throughput imaging-based multiplex screening for AR functional analyses, Szafran et al. also demonstrated a unique response pattern in the presence of synthetic AR ligand methyltrienolone (R1881) [21]. In combination with $10 \mathrm{nM}$ R1881, D caused an antagonistic response (45\% decrease in AR transcriptional reporter gene activity, $\left.\mathrm{EC}_{50}=13.2 \mu \mathrm{M}\right)$. However, it decreased R1881- induced nuclear translocation $\left(95 \%, \mathrm{EC}_{50}=12.3 \mu \mathrm{M}\right)$. The small nuclear pool also exhibited a loss of hyperspeckling $\left(94 \%\right.$ in NVAR, $\left.\mathrm{EC}_{50}=10.6 \mu \mathrm{M}\right)$ [21]. These findings further suggested $\mathrm{AR}$ as one target in the in vitro anti-prostate cancer activity of D and DA.

Others reported mechanisms other than AR suppression in the anti-prostate cancer effect of D. Yim et al. reported that $D$ $(25-100 \mu \mathrm{M})$ inhibited the cell growth in human PCA cells DU145, PC-3 (both lines are AR-negative), and LNCaP [22]. The D-induced growth inhibition was associated with a strong $\mathrm{G}_{1}$ arrest in DU145 and LNCaP cells and $\mathrm{G}_{1}, \mathrm{~S}$ as well as $\mathrm{G}_{2}-$ $\mathrm{M}$ arrests, depending upon doses and treatment times in PC-3 cells. Interestingly, non-cancerous "normal" human prostate epithelial PWR-1E cells were moderately growth arrested but was not killed. This indicated that D might offer some advantage in drug selectivity [22]. Another study reported that D suppressed the $\mathrm{Wnt} / \beta$-catenin pathway in PC-3 cells [23]. In this cell model, D promoted $\beta$-catenin degradation and suppressed the expression of the related downstream factors cyclin D1 and c-myc. Unlike D, DOH had no effect on intracellular level of $\beta$-catenin and PC-3 proliferation. More recently, Choi et al. reported that $\mathrm{D}$ induced apoptosis in primary malignant tumor-derived human prostate cells, RC-58T/h/SA\#4, and investigated the potential mechanisms [24]. They found RC-58T/h/SA\#4 cells slightly more sensitive to $\mathrm{D}$ than human prostate epithelial RWPE-1 cells. Treatment of the RC-58T/h/ SA\#4 cells with 80 and $100 \mu \mathrm{M}$ D for $48 \mathrm{~h}$ significantly induced apoptosis, evidenced by nuclear condensation, DNA fragmentation, PARP cleavage, and increased caspase activities.

It is noteworthy that the cellular events mentioned in these papers were observed when cells were treated with $\mathrm{D}$ at the concentrations much higher (10-50 fold) than that have been reported for AR suppressing effect $[19,20]$. As will be discussed in pharmacokinetics sections later, even single micromolar levels of D or DA will be not likely achievable in rodents or humans by oral route of intake.

\section{Lung Cancer}

In a study using mouse Lewis lung cancer (LLC) model, daily administration of D of $4 \mathrm{mg} / \mathrm{kg}$ by i.p. injection for 3 weeks reduced tumor growth by $65 \%$ [25]. Tumor microvessel density was dramatically lower in D-treated group as evidenced by CD31 immunohistochemistry staining. The expression levels of phospho-VEGFR2, p-ERK (extracellular signal-regulated kinase), p-JNK (c-Jun N-terminal kinase), and matrix metalloproteinase (MMP)-2 were also significantly reduced compared with control tumors. These observations suggested that an anti-angiogenesis effect of D (or metabolites) might contribute to its tumor suppression efficacy [25]. We also evaluated the effect of D and DOH in the LLC allograft model [8]. In that study, $50 \mathrm{mg} / \mathrm{kg}$ D or DOH was administered daily 
in $1 \%$ Tween 80 by gavage for 13 consecutive days starting at 5 days after LLC tumor inoculation. Tumor volume and weight were suppressed by about $40 \%$ by either D or DOH. Though the in vitro activities of $\mathrm{D}$ and $\mathrm{DOH}$ were different as we and others have reported for prostate and breast cancer cells $[20,22,26]$, the data suggest that orally administrated $\mathrm{D}$ and DOH were equally efficacious against LLC allograft.

\section{Colon Cancer}

In murine colon carcinoma CT-26 cells, even though 10 to $20 \mu \mathrm{M}$ D and DOH marginally reduced the viability and proliferation of CT-26 cells in vitro, both compounds substantially inhibited their invasive activity [27]. DOH exhibited a stronger anti-invasive potential than D. Mechanistic studies indicated that $\mathrm{D}$ and $\mathrm{DOH}$ suppressed both the expression and activities of MMP-2 and MMP-9, the latter being more important for the invasion of CT-26 cells. Phosphorylation of ERK and JNK, which played a critical role for the invasion of CT26 cells through modulating the expression of MMP-9, was found to be downregulated by $\mathrm{D}$ and $\mathrm{DOH}$ treatment. In addition, daily oral administration of D and DOH $(10 \mathrm{mg} / \mathrm{kg}$ for 14 days) starting $30 \mathrm{~min}$ prior to the injection of cancer cells through tail vein reduced the formation of tumor nodules in the lungs and decreased lung weight caused by CT-26 metastases. The data suggested that $\mathrm{DOH}$ and $\mathrm{D}$ (most probably through conversion to $\mathrm{DOH}$, see pharmacokinetic sections) might be anti-metastatic agents targeting MMPs and their upstream signaling molecules [27].

\section{Sarcoma}

In a mouse allograft study, D or DA, at doses of 50 and $100 \mathrm{mg} / \mathrm{kg}$ by i.p. injection daily for 9 days, suppressed Sarcoma-180 tumor growth in ICR mice [28]. The authors also showed that D or DA each was able to extend the life span of mice inoculated Sarcoma-180 tumor cells by i.p. injection: the median survival times were 32.3 and 34.2 days for 50 and $100 \mathrm{mg} / \mathrm{kg}$ DA-treated groups, respectively, and were 29.0 and 39.6 days for 50 and $100 \mathrm{mg} / \mathrm{kg}$ D-treated groups, respectively, compared with the median survival time for the control group of just 22.4 days [28].

\section{Anti-angiogenesis Effects}

In addition to cancer cell models, it has been reported that $\mathrm{D}$ and DOH at non-cytotoxic doses $(1-20 \mu \mathrm{M})$ reduced VEGFinduced cell proliferation, migration, and capillary tube formation of human umbilical vein endothelial cells (HUVEC) [29]. The anti-angiogenic effect by $\mathrm{D}$ and $\mathrm{DOH}$ was possibly associated with suppression of ERK and JNK activation. Additionally, D and DOH suppressed microvessel formation on chorioallantoic membranes in fertilized eggs and in mouse
Matrigel plugs. Moreover, orally administered D and DOH inhibited VEGF-induced angiogenesis in Matrigel plugs [29]. Others reported similar results about anti-angiogenesis effect with D and DA in association with inhibition of VEGFinduced phosphorylation of VEGFR-2, extracellular signalregulated kinases, c-Jun N-terminal kinase, and mitogenactivated protein kinases and suppression by D of LLC lung allograft tumor growth in vivo [25].

\section{Anti-cancer Activities of Chemicals in AGN Other Than D/DA}

We reviewed other chemicals other than D and DA isolated from AGN that have been evaluated by the NCI Developmental Therapeutics Program for the in vivo ability to increase survival of allograft tumor-bearing mice (http://dtp.nci.nih. gov/docs/dtp_search.html) [1•]. Xanthotoxin might afford a reasonable anti-cancer activity in $25-64 \mathrm{mg} / \mathrm{kg}$ dosage range that increased survival time of the treated mice. Its doselimiting lethality was at $256 \mathrm{mg} / \mathrm{kg}$. Scopoletin was about one tenth as potent for anti-cancer $(400 \mathrm{mg} / \mathrm{kg})$, without detectable lethality to the host mice. Marmesin, umbelliferone, and bergapten treatment did not result in a survival advantage in the dosage ranges used. A recent paper indicated that five chemicals isolated form AGN including marmesinin, nodakenin, skimmin, apiosylskimmin, and magnolioside showed no significant cytotoxicity $\left(\mathrm{IC}_{50}>100 \mu \mathrm{M}\right)$ toward SK-OV-3 ovarian cancer cell line in vitro, though marmesinin and magnolioside exerted notable anti-plasmodial activity against chloroquine-sensitive strains of Plasmodium falciparum with $\mathrm{IC}_{50}$ of 5.3 and $8.2 \mu \mathrm{M}$, respectively [30].

Angelan is a $10-\mathrm{kDa}$ polysaccharide purified from AGN, composed of arabinose, galactose, and galacturonic acid but does not contain glucose [31]. It has been reported to lengthen the survival time of B16F10 tumor-bearing mice when i.p. administered at $30 \mathrm{mg} / \mathrm{kg}$ [32]. The primary targets of angelan were assumed to be macrophages and natural killer (NK) cells which were involved in nonspecific immunity [31, 32]. Angelan markedly induced transcription of inducible nitric oxide synthase (iNOS), interleukin-1 $\beta$ (IL-1 $\beta$ ), and tumor necrosis factor $\alpha$ (TNF- $\alpha)$ in murine macrophage RAW 264.7 cells, which was mediated by specific activation of NF-KB/Rel [33, 34]. Another study claimed the macrophage activation by angelan differed from that induced by bacterial lipopolysaccharide (LPS) without strong data [35]. Further studies indicated that angelan induced NF- $\mathrm{kB} /$ Rel activation through CD14 and complement receptor type 3 (CR3) membrane receptor and p38 kinase; the latter is critical for the signal transduction leading to NF- $\mathrm{KB} /$ Rel activation in murine macrophages [36]. Later on, the same group investigated the effects of angelan on dendritic cell (DC) maturation and found a crucial role of TLR4 signaling pathways [37]. These results 
suggested the possible use of angelan in DC-based immunotherapies. However, the alcoholic extract of AGN sold as dietary supplements should contain little angelan, if any.

\section{Pharmacokinetics of D, DA, and DOH in Rodent Models}

Although D and DA are mainly responsible for the cancer cell arrest actions in cell culture models, available rodent studies have shown a rapid conversion of D and DA to $\mathrm{DOH}$, casting grave doubts over the in vivo mechanistic relevance of $\mathrm{D}$ and DA. Park et al. detected DOH $(\sim 80 \mu \mathrm{g} / \mathrm{ml})$ in the plasma of rats after administration of $300 \mathrm{mg} / \mathrm{kg}$ D or the methanolic or ether extract of AGN while detecting no D [38]. We detected $\mathrm{DOH}$ as the major metabolite after i.p. injection and failed to detect the parent compounds in plasma [8]. We then systematically evaluated a method for the simultaneous analysis of D/DA and DOH in biological matrixes by HPLC and applied the method to the detection of plasma or tissue levels of these analytes in several in vivo studies [39]. In male C57BL/6 mice given a single dose of $4.8 \mathrm{mg} \mathrm{D} / \mathrm{DA}(\sim 240 \mathrm{mg} / \mathrm{kg})$ either by gavage or i.p. injection, we detected that plasma concentrations for D/DA and DOH were 11.2 and $79.7 \mu \mathrm{g} / \mathrm{mL}$, respectively, when $\mathrm{D} / \mathrm{DA}$ was administered via i.p. injection, and 0.54 and $14.9 \mu \mathrm{g} / \mathrm{mL}$ when given by gavage. D/DA and DOH content in the tumor tissues from nude mouse xenografts correlated very well with that in plasma. Overall, our results affirm the conclusion that majority of D and DA converts to $\mathrm{DOH}$ in rodents with a tiny fraction remaining as the parent compounds.

The specific organ site(s) for the conversion of D and DA is likely the liver by first pass metabolism when they are administrated by routes other than i.v. infusion, as demonstrated recently [40 ${ }^{\bullet}$. Plasma $\mathrm{D}$ decreased rapidly after i.v. administration with an initial half-life of $0.05 \mathrm{~h}$ and was not detectable after $1 \mathrm{~h}$. The concentration of DOH increased rapidly, reaching peak at $t_{\max }=0.25 \mathrm{~h}$, and the area under the plasma concentration-time curve $\left(\mathrm{AUC}_{\mathrm{DOH}}\right)$ was 4.3 -folds of $\mathrm{AUC}_{\mathrm{D}}$. In portal vein-cannulated rats, $D$ was the major pyranocoumarin in blood collected from the portal vein after gavage, whereas DOH was the dominant chemical in blood circulation if $\mathrm{D}$ was administered through the portal vein with the fraction of dose escaping the liver extremely low (FH= $0.11)$.

Absorption of DOH itself in rats was found quite efficient [41]: following i.v. administration, non-linear elimination of DOH was observed; whereas after oral administration, $\mathrm{DOH}$ exhibited rapid absorption $\left(t_{\max } 0.4-0.9 \mathrm{~h}\right)$, resulting in bioavailability of $\sim 45 \%$ ( $\mathrm{AUC}_{\text {oral }} / \mathrm{AUC}_{\mathrm{iv}}$ ). Following up our mouse study, we compared the plasma profiles of $\mathrm{DOH}$, when equimolar D/DA or DOH was given to rats by gavage [42]. $\mathrm{DOH}$ gavage led to a faster attainment of plasma $\mathrm{DOH}$ peak $\left(t_{\max } \sim 0.7 \mathrm{~h}\right)$ and much higher peak levels than an equimolar amount of D/DA mixture or as AGN ethanol extract, resulting in 2.4-folds higher AUC (65,012 vs. $27,033 \mathrm{~h} \mathrm{ng} / \mathrm{mL}$ for $\mathrm{DOH}$ and D/DA groups, respectively). We detected peak levels of D and DA in the plasma of rats administered with D/DA or AGN extract only in the nanomolar range $\left(t_{\max } \sim 0.5 \mathrm{~h}\right)$ with a more sensitive UHPLC-MS/MS method.

Contradicting the above, a Korean group reported that both $\mathrm{D}$ and DA were detectable at high concentrations in rat plasma up to $72 \mathrm{~h}$ after the D/DA mixture was administered by either gavage (200 mg/rat) or i.v. injection ( $2 \mathrm{mg} / \mathrm{rat})$ [43]. There are many troubling aspects of this study including the near-total absorption of the gavage dose.

In summary, the structural difference between $\mathrm{DOH}$ and D/ DA is obvious and the natural abundance of DOH in AGN is much lower than D and DA, if at all. We and others documented the different in vitro biological activities of DOH compared to D/DA such as anti-AR and AChE inhibitory effect $[20,44]$. On the other hand, others have reported similar in vitro anti-angiogenesis and neuro-protective effects of D, DA, and DOH $[25,29,45]$. It is likely that $\mathrm{DOH}$ and/or its further metabolite(s) are the real in vivo "effector" for the many observed effects, i.e., D and DA act as natural prodrugs of $\mathrm{DOH}$.

\section{Pharmacokinetics of D and DA in Humans}

Given the first pass liver metabolism in rodents, we compared the metabolic rate of D and DA using liver microsomes from humans and rodents and identified potential metabolites of $\mathrm{D}$ and DA in human liver microsomal incubations using LCMS/MS [46 •]. In murine and human liver microsomal incubations in the absence of NADPH, D was efficiently hydrolyzed to DOH presumably by hepatic esterases, but DA was not (see proposed metabolism schema in Fig. 1b). In contrast, formation of DOH from DA was mediated mainly by cytochrome P450(s) requiring NADPH. The human liver S9 fractions metabolized both D and DA more slowly than those of the mouse. We identified nine potential metabolites of $\mathrm{D}$ and nine metabolites of DA from human liver microsomal incubations using a hybrid triple quadruple linear ion trap LC-MS/ MS system, and many of them were later verified to be also present in plasma samples from rodent PK studies. In contrast, D and DA were not hydrolyzed to DOH with rat intestinal S9 microsomal in vitro metabolism assay with or without NADP $\mathrm{H}[42]$.

To address a key question for human translatability of animal model studies of D and DA or AGN extract, we conducted a single oral dose human PK study of D and DA delivered through an AGN-based dietary supplement Cogni.Q (purchased from Quality of Life Labs, Purchase, NY) in 10 healthy men and 10 healthy women, each consuming 
$119 \mathrm{mg}$ D and $77 \mathrm{mg}$ DA from four vegicaps [47]. Analyses of plasma using UHPLC-MS/MS showed mean time to peak concentration $\left(t_{\max }\right)$ of $2.1,2.4$, and $3.3 \mathrm{~h}$ and mean peak concentration $\left(C_{\max }\right)$ of $5.3,48.1$, and $2480 \mathrm{nmol} / \mathrm{L}$ for $\mathrm{D}$, $\mathrm{DA}$, and $\mathrm{DOH}$, respectively. The terminal elimination halflife $\left(t_{1 / 2}\right)$ for D and DA was similar (17.4 and $19.3 \mathrm{~h}$ ) and each was much longer than that of $\mathrm{DOH}(7.4 \mathrm{~h})$. The mean $\mathrm{AUC}_{0-}$ $48 \mathrm{~h}$ for D, DA, and DOH was estimated as 37,335 , and 27 , $579 \mathrm{~h} \mathrm{nmol} / \mathrm{L}$, respectively. Gender-wise, men absorbed the parent compounds faster and took shorter time to reach $\mathrm{DOH}$ peak concentration. The human data supported an extensive conversion ( $>97 \%$ ) of D and DA to DOH, even though they metabolized DA slightly slower than rodents. Therefore, the data generated in rodent models concerning anti-cancer efficacy, safety, tissue distribution, and pharmacodynamic biomarkers will likely be relevant for human translation.

\section{Conclusions and Future Research Directions}

PK studies in rodent models and in humans have provided unequivocal evidence that D and DA from dietary supplements or enteral route are extensively converted to $\mathrm{DOH}$ by first pass metabolism in the liver, resulting in very low levels of these parent compounds in peripheral circulation (Fig. 1b). Such insights cast grave doubt over the relevance of the "mechanisms" of D and DA in cell culture studies. In a primary carcinogenesis prevention study, we have observed that gavage-administered equimolar D/DA does not fully account for the preventive efficacy of AGN extract on TRAMP neuroendrocrine carcinomas, in spite of equal inhibitory action on the TRAMP prostate epithelial lesions.

Therefore, in the near future, the following should be addressed.

1. Given that DOH has been identified unequivocally as the major and rapid metabolite of D and DA in rodents and humans, experiments are needed to compare its in vivo efficacy with the parent compounds at PK-bioequivalent dosages to determine how much these pyranocoumarins contribute to anti-cancer activities of AGN.

2. Knowledge of DOH further metabolism should be obtained. DOH has been reported to exhibit high stability to oxidative and glucoronic metabolism in human and rat liver microsomes in vitro [41]. The in vivo DOH metabolites should be investigated. Since CYP450 is implicated in DA and, to less extent, D hepatic conversion to DOH through oxygenated intermediates [46•, 48] (Fig. 1b), the extent and contribution of these metabolic intermediates should not be overlooked nor the impact and interactions with CYP450 inhibitor drugs.
3. The pharmacodynamic targets of $\mathrm{DOH}$ and its metabolites should be identified, in cancer cells and cells that make up the tumor microenvironment. Our human PK data further motivate such endeavor because the likelihood for D and $\mathrm{DA}$ to directly bind proteins such as AR and AChE associated with anti-cancer and neuro-protective effects would be extremely low, if not nil. So far, Kang et al. has identified a few DOH-binding proteins from brain tissue by affinity chromatography and mass spectrometry [49]. They include heat shock protein $90-\mathrm{kDa}$ beta member 1 (GRP94 or HSP90B1), enolase 1 (ENO1), actin gamma 1 (ACTG1), heterogeneous nuclear ribonucleoprotein A2/ B1 (HNRNPA2B1), and non-muscle myosin heavy polypeptide 9 (MYH9). The search strategy might be applicable to cancer and other cell types in the cancer microenvironments.

4. Besides pyranocoumarins, other components in AGN extract should be investigated. Whether these low abundant chemicals in AGN mediate or work in synergy with D/DA/DOH to exert anti-cancer activity remain an open question.

Acknowledgments Supported by National Center for Complementary and Integrative Health (NCCIH/NCCAM) grants R01AT007395 (Lü, Xing) and R21AT005383 (Lü).

\section{Compliance with Ethics Guidelines}

Conflict of Interest Junxuan Lü, Jinhui Zhang, Li Li, Cheng Jiang, and Chengguo Xing declare that they have no conflict of interest.

Human and Animal Rights and Informed Consent This article does not contain any studies with human or animal subjects performed by any of the authors.

\section{References}

Papers of particular interest, published recently, have been highlighted as:

- Of importance

•- Of major importance

1. Zhang J, Li L, Jiang C, Xing C, Kim SH, Lu J. Anti-cancer and other bioactivities of Korean Angelica gigas Nakai (AGN) and its major pyranocoumarin compounds. Anti Cancer Agents Med Chem. 2012;12:1239-54. This paper provided a comprehensive and critical review of anti-cancer and other bioactivities of AGN and its phytochemicals. It also identified critical gaps of knowledge and future research needs.

2. Sarker SD, Nahar L. Natural medicine: the genus Angelica. Curr Med Chem. 2004;11:1479-500.

3. Zhao KJ, Dong TT, Tu PF, Song ZH, Lo CK, Tsim KW. Molecular genetic and chemical assessment of radix Angelica (Danggui) in China. J Agric Food Chem. 2003;51:2576-83.

4. Kim MR, Abd El-Aty AM, Choi JH, Lee KB, Shim JH. Identification of volatile components in Angelica species using 
supercritical- $\mathrm{CO}_{2}$ fluid extraction and solid phase microextraction coupled to gas chromatography-mass spectrometry. Biomed Chromatogr:BMC. 2006;20:1267-73.

5. Kim MR, Abd El-Aty AM, Kim IS, Shim JH. Determination of volatile flavor components in danggui cultivars by solvent free injection and hydrodistillation followed by gas chromatographicmass spectrometric analysis. J Chromatogr A. 2006;1116:259-64.

6. Piao XL, Park JH, Cui H, Kim DH, Yoo HH. Development of gas chromatographic mass spectrometry-pattern recognition method for the quality control of Korean Angelica. J Pharm Biomed Anal. 2007;44:1163-7.

7. Kim EJ, Kwon J, Park SH, Park C, Seo YB, Shin HK, et al. Metabolite profiling of Angelica gigas from different geographical origins using ${ }^{1} \mathrm{H}$ NMR and UPLC-MS analyses. J Agric Food Chem. 2011;59:8806-15.

8. Lee HJ, Lee EO, Lee JH, Lee KS, Kim KH, Kim SH, et al. In vivo anti-cancer activity of Korean Angelica gigas and its major pyranocoumarin decursin. Am J Chin Med. 2009;37:127-42.

9.• Zhang J, Wang L, Zhang Y, Li L, Tang S, Xing C, Kim SH, Jiang C, $\mathrm{Lu}$ J. Chemopreventive effect of Korean Angelica root extract on TRAMP carcinogenesis and integrative "omic" profiling of affected neuroendocrine carcinomas. Mol Carcinog 2014. doi: 10.1002/ mc.22230. This paper reported for the first time preventive efficacy of AGN on a primary carcinogenesis model. The omics profiling suggested multiple targeting actions not only in cancer cells, but also their microenvironments.

10. Bullard RS, Gibson W, Bose SK, Belgrave JK, Eaddy AC, Wright $\mathrm{CJ}$, et al. Functional analysis of the host defense peptide human beta defensin-1: new insight into its potential role in cancer. Mol Immunol. 2008;45:839-48.

11. Kela I, Harmelin A, Waks T, Orr-Urtreger A, Domany E, Eshhar Z. Interspecies comparison of prostate cancer gene-expression profiles reveals genes associated with aggressive tumors. Prostate. 2009;69: 1034-44.

12. Haram KM, Peltier HJ, Lu B, Bhasin M, Otu HH, Choy B, et al. Gene expression profile of mouse prostate tumors reveals dysregulations in major biological processes and identifies potential murine targets for preclinical development of human prostate cancer therapy. Prostate. 2008;68:1517-30.

13. Zhang J, Wang L, Zhang Y, Li L, Higgins L, Lu J. Lobe-specific proteome changes in the dorsal-lateral and ventral prostate of TRAMP mice versus wild-type mice. Proteomics. 2011;11:2542-9.

14. Yip PY, Kench JG, Rasiah KK, Benito RP, Lee CS, Stricker PD, et al. Low AZGP1 expression predicts for recurrence in marginpositive, localized prostate cancer. Prostate. 2011;71:1638-45.

15. Henshall SM, Horvath LG, Quinn DI, Eggleton SA, Grygiel JJ, Stricker PD, et al. Zinc-alpha2-glycoprotein expression as a predictor of metastatic prostate cancer following radical prostatectomy. $\mathrm{J}$ Natl Cancer Inst. 2006;98:1420-4

16. Pittoni P, Tripodo C, Piconese S, Mauri G, Parenza M, Rigoni A, et al. Mast cell targeting hampers prostate adenocarcinoma development but promotes the occurrence of highly malignant neuroendocrine cancers. Cancer Res. 2011;71:5987-97.

17. Morgenbesser SD, McLaren RP, Richards B, Zhang M, Akmaev VR, Winter SF, et al. Identification of genes potentially involved in the acquisition of androgen-independent and metastatic tumor growth in an autochthonous genetically engineered mouse prostate cancer model. Prostate. 2007;67:83-106

18. Tang S, Zhang Y, Jiang P, Zhang J, Xing C, Kim S-H, Jiang C, Lü J. A paradigm of carcinogenesis lineage specificities of cancer chemoprevention: Korean Angelica and its pyranocoumarins in the transgenic adenocarcinoma of mouse prostate model. Proc AACR 2013. Abstract \#LB-184. doi:10.1158/1538-7445.AM2013-LB184

19. Jiang C, Lee HJ, Li GX, Guo JM, Malewicz B, Zhao Y, et al. Potent antiandrogen and androgen receptor activities of an Angelica gigas- containing herbal formulation: identification of decursin as a novel and active compound with implications for prevention and treatment of prostate cancer. Cancer Res. 2006;66:453-63.

20. Guo JM, Jiang C, Wang Z, Lee HJ, Hu HB, Malewicz B, et al. A novel class of pyranocoumarin anti-androgen receptor signaling compounds. Mol Cancer Ther. 2007;6:907-17.

21. Szafran AT, Szwarc M, Marcelli M, Mancini MA. Androgen receptor functional analyses by high throughput imaging: determination of ligand, cell cycle, and mutation-specific effects. PLoS One. 2008;3:e3605.

22. Yim D, Singh RP, Agarwal C, Lee S, Chi H, Agarwal R. A novel anticancer agent, decursin, induces $\mathrm{G}_{1}$ arrest and apoptosis in human prostate carcinoma cells. Cancer Res. 2005;65:1035-44.

23. Song GY, Lee JH, Cho M, Park BS, Kim DE, Oh S. Decursin suppresses human androgen-independent $\mathrm{PC}_{3}$ prostate cancer cell proliferation by promoting the degradation of beta-catenin. Mol Pharmacol. 2007;72:1599-606.

24. Choi SR, Lee JH, Kim JY, Park KW, Jeong IY, Shim KH, Lee MK, Seo KI. Decursin from Angelica gigas Nakai induces apoptosis in RC-58T/h/SA\#4 primary human prostate cancer cells via a mitochondria-related caspase pathway. Food Chem Toxicol 2011;49:2517-23

25. Jung MH, Lee SH, Ahn EM, Lee YM. Decursin and decursinol angelate inhibit VEGF-induced angiogenesis via suppression of the VEGFR-2-signaling pathway. Carcinogenesis. 2009;30:65561 .

26. Jiang C, Guo JM, Wang Z, Xiao BX, Lee HJ, Lee EO, Kim SH, Lu JX. Decursin and decursinol angelate inhibit estrogen-stimulated and estrogen-independent growth and survival of breast cancer cells. Breast Cancer Res. 2007; 9.

27. Son SH, Park KK, Park SK, Kim YC, Kim YS, Lee SK, et al. Decursin and decursinol from angelica gigas inhibit the lung metastasis of murine colon carcinoma. Phytother Res. 2011;25:95964.

28. Lee S, Lee YS, Jung SH, Shin KH, Kim BK, Kang SS. Anti-tumor activities of decursinol angelate and decursin from Angelica gigas. Arch Pharm Res. 2003;26:727-30.

29. Son SH, Kim MJ, Chung WY, Son JA, Kim YS, Kim YC, et al. Decursin and decursinol inhibit VEGF-induced angiogenesis by blocking the activation of extracellular signal-regulated kinase and c-Jun N-terminal kinase. Cancer Lett. 2009;280:86-92.

30. Moon HI, Lee JH, Lee YC, Kim KS. Antiplasmodial and cytotoxic activity of coumarin derivatives from dried roots of Angelica gigas Nakai in vitro. Immunopharmacol Immunotoxicol. 2011;33:663-6.

31. Han SB, Kim YH, Lee CW, Park SM, Lee HY, Ahn KS, et al. Characteristic immunostimulation by angelan isolated from Angelica gigas Nakai. Immunopharmacology. 1998;40:39-48.

32. Han SB, Lee CW, Kang MR, Yoon YD, Kang JS, Lee KH, et al. Pectic polysaccharide isolated from Angelica gigas Nakai inhibits melanoma cell metastasis and growth by directly preventing cell adhesion and activating host immune functions. Cancer Lett. 2006;243:264-73.

33. Jeon YJ, Han SB, Ahn KS, Kim HM. Activation of NF-kappaB/Rel in angelan-stimulated macrophages. Immunopharmacology. 1999;43:1-9.

34. Jeon YJ, Han SB, Lee SH, Kim HC, Ahn KS, Kim HM. Activation of mitogen-activated protein kinase pathways by angelan in murine macrophages. Int Immunopharmacol. 2001;1:237-45.

35. Jeon YJ, Han SB, Ahn KS, Kim HM. Differential activation of murine macrophages by angelan and LPS. Immunopharmacology. 2000;49:275-84.

36. Jeon YJ, Kim HM. Experimental evidences and signal transduction pathways involved in the activation of NF-kappa B/Rel by angelan in murine macrophages. Int Immunopharmacol. 2001;1:1331-9.

37. Kim JY, Yoon YD, Ahn JM, Kang JS, Park SK, Lee K, et al. Angelan isolated from Angelica gigas Nakai induces dendritic cell 
maturation through toll-like receptor 4. Int Immunopharmacol. 2007;7:78-87.

38. Park RJ, Kim NJ, Lee KT, Seo SH. Comparative studies on concentration of decursinol in plasma after oral administration of Angelicae Gigantis Radix extract and combined use of Decursin and Cnidii Rhizoma Extract or Bupleuri Radix Extract in rats. Korean J Pharmacogn. 2001;32:72-8.

39. Li L, Zhang J, Shaik AA, Zhang Y, Wang L, Xing C, et al. Quantitative determination of decursin, decursinol angelate, and decursinol in mouse plasma and tumor tissue using liquid-liquid extraction and HPLC. Planta Med. 2012;78:252-9.

40. Park HS, Kim B, Oh JH, Kim YC, Lee YJ. First-pass metabolism of decursin, a bioactive compound of Angelica gigas, in rats. Planta Med. 2012;78:909-13. This paper carefully evaluated the role of liver to metabolize decursin using portal vein-canulated rat model. It provided critical experimental evidence of rapid first pass conversion of D to DOH.

41. Song JS, Chae JW, Lee KR, Lee BH, Choi EJ, Ahn SH, et al. Pharmacokinetic characterization of decursinol derived from Angelica gigas Nakai in rats. Xenobiotica. 2011;41:895-902.

42. Li L, Zhang J, Xing C, Kim SH, Lu J. Single oral dose pharmacokinetics of decursin, decursinol angelate, and decursinol in rats. Planta Med. 2013;79:275-80.

43. Kim KM, Kim MJ, Kang JS. Absorption, distribution, metabolism, and excretion of decursin and decursinol angelate from Angelica gigas Nakai. J Microbiol Biotechnol. 2009;19:1569-72.
44. Kang SY, Lee KY, Sung SH, Park MJ, Kim YC. Coumarins isolated from Angelica gigas inhibit acetylcholinesterase: structure-activity relationships. J Nat Prod. 2001;64:683-5.

45. Kang SY, Kim YC. Decursinol and decursin protect primary cultured rat cortical cells from glutamate-induced neurotoxicity. J Pharm Pharmacol. 2007;59:863-70.

46. Li L, Zhang J, Xing C, Kim SH, Jiang C, Lu J. In vitro metabolism of pyranocoumarin isomers decursin and decursinol angelate by liver microsomes from man and rodents. Planta Med. 2013;79: 1536-44. This paper used human liver microsomal systems to compare with murine counterparts to evaluate simiarities for hepatic metabolism of D and DA across species. Provided structural information on potential human liver intermediate $D$ and DA metabolites leading to DOH and differential involvement of cytochrome p450 and esterases for the conversion.

47. Zhang J, Li L, Hale TW, Chee W, Xing C, Jiang C, Lü J. Single oral dose pharmacokinetics of decursin and decursinol angelate in healthy adult men and women. PLoS One. 2015;10;e0114992. doi:10.1371/journal.pone.0114992.

48. Yoo HH, Lee MW, Kim YC, Yun CH, Kim DH. Mechanism-based inactivation of cytochrome $\mathrm{p} 4502 \mathrm{~A} 6$ by decursinol angelate isolated from Angelica gigas. Drug Metab Dispos. 2007;35:1759-65.

49. Kang HJ, Yoon TS, Jeong DG, Kim Y, Chung JW, Ha JS, et al. Identification of proteins binding to decursinol by chemical proteomics. J Microbiol Biotechnol. 2008;18:1427-30. 